\title{
How will we close the gap in smoking rates for pregnant Indigenous women?
}

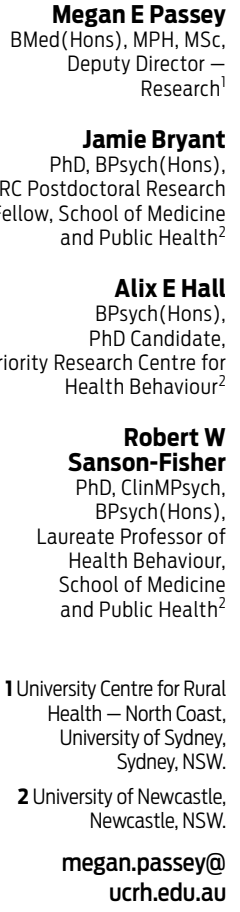

doi: 10.5694/mjal2.11848 ntenatal smoking is the most important modifiable cause of adverse pregnancy outcomes. ${ }^{1}$ Indigenous Australian women are more than three times more likely to smoke during pregnancy than nonIndigenous women. ${ }^{2}$ As a result, adverse outcomes are more frequent in Indigenous than non-Indigenous babies, with smoking as an independent risk factor. ${ }^{3}$

Reviews of antenatal smoking interventions have shown effective cessation strategies for pregnant women. ${ }^{1}$ However, persistently high rates of smoking during pregnancy among Indigenous women suggest that current interventions have had limited impact. Finding ways to effectively reduce smoking in pregnant Indigenous populations is a high priority. Previous systematic reviews have examined smoking cessation interventions for Indigenous peoples; however, none has specifically investigated smoking cessation among pregnant Indigenous women. ${ }^{4,5}$

We undertook a systematic review to examine the effectiveness and methodological quality of smoking cessation interventions targeting pregnant Indigenous women. In December 2012 we searched MEDLINE, PsycINFO, CINAHL (Cumulative Index to Nursing and Allied Health Literature) and Cochrane databases with appropriate search terms, and checked reference lists of retrieved articles. Papers were included if they reported a smoking cessation intervention aimed at pregnant Indigenous women, included a control group and provided cessation results specifically for pregnant Indigenous women. Only peer-reviewed, English-language papers were included. We extracted data and assessed methodological quality against Effective Practice and Organisation of Care quality criteria. ${ }^{6}$

Of 59 identified papers only two met eligibility criteria: one from the United States with Alaskan Native women, ${ }^{7}$ and one from Australia with Aboriginal and Torres Strait Islander women. ${ }^{8}$ Both involved culturally tailored interventions specifically developed for the target group, and used face-to-face counselling, structured follow-up, attempts to involve family members and nicotine replacement therapy (NRT). Both studies found no treatment effect and had a number of limitations (Box).

This lack of evidence of effective smoking cessation interventions for pregnant Indigenous women prevents implementation of evidence-based programs and highlights a critical need for methodologically rigorous testing of possible strategies.

\section{What interventions should we test?}

Evidence from research with Indigenous populations, and with pregnant women generally, provides guidance about the strategies that hold promise for pregnant Indigenous women. These strategies are outlined as follows.

\begin{abstract}
Summary
- Aboriginal and Torres Strait Islander women are more than three times more likely to smoke during pregnancy than non-Indigenous women, greatly increasing the risk of poor birth outcomes.

- Our systematic review found that there is currently no evidence for interventions that are effective in supporting pregnant Aboriginal and Torres Strait Islander women to quit smoking, which impedes development and implementation of evidence-informed policy and practice.

- There is an urgent need for methodologically rigorous studies to test innovative approaches to addressing this problem.
\end{abstract}

\section{Tailor interventions to local culture}

Interventions for Indigenous people need to be culturally secure and locally tailored in order to increase acceptability and accessibility. ${ }^{4,5,9}$ Involving local people in developing and tailoring intervention resources to the local context is critical for improving cultural appropriateness, building ownership and enhancing a sense of autonomy, all of which are important in successful cessation. ${ }^{10}$

\section{Include routine assessment and support}

Smoking cessation guidelines for pregnant women recommend a systematic approach to cessation where every woman is asked about her smoking status, with smokers followed up and supported to quit in a respectful manner. ${ }^{11}$ Health professionals may be reluctant to repeatedly assess smoking status due to concerns that it may be deleterious to their relationship with women and the women's engagement with care., ${ }^{92-14}$ However, most Indigenous women expect antenatal care to include smoking cessation advice. ${ }^{15}$ Systems to support routine assessment and support should be included in intervention trials.

\section{Provide relevant information}

Indigenous women's knowledge of specific risks of smoking while pregnant is often vague. ${ }^{9,15,16}$ Providing information on the harms of smoking and benefits of cessation may motivate some women to attempt to quit. Discussing the woman's role as a mother and a role model for her family may be more motivating for some Indigenous women than health risk narratives and should be addressed in intervention trials.

\section{Deliver cessation support through all antenatal providers}

Overall, $78 \%$ of Indigenous women attend five or more antenatal visits during their pregnancies. ${ }^{2}$ Providing cessation support through routine antenatal care 


\begin{tabular}{|c|c|c|}
\hline Criteria & Patten et $\mathrm{al}^{7}$ & Eades et $\mathrm{al}^{8}$ \\
\hline Design & Clinical controlled trial & Randomised controlled trial \\
\hline Allocation sequence adequately generated? & Unclear & Low risk \\
\hline Concealment of allocation? & Unclear & Low risk \\
\hline Baseline outcome measurements similar? & Low risk & Unclear \\
\hline Baseline characteristics similar? & Low risk & Low risk \\
\hline Incomplete outcome data adequately addressed? & Unclear & Low risk \\
\hline Knowledge of allocated interventions prevented? & Unclear & Unclear \\
\hline Protection against contamination? & High risk & High risk \\
\hline Selective outcome reporting? & Low risk & Low risk \\
\hline Free from other risk of bias? & High risk & Low risk \\
\hline Comments & $\begin{array}{l}\text { The low consent rate and the fact that many } \\
\text { women did not take part because they were } \\
\text { not ready to quit increases the chances of } \\
\text { selection bias. The focus of the study was on } \\
\text { feasibility and acceptability rather than on } \\
\text { outcomes, although outcomes are reported }\end{array}$ & $\begin{array}{c}\text { High loss to follow-up ( } 33 \% \text { ), but this did not differ } \\
\text { between the groups. Randomisation was by week of } \\
\text { first visit, so the staff who were recruiting the women } \\
\text { were aware of the group allocation. This may have } \\
\text { contributed to the greater numbers recruited to } \\
\text { the intervention group }\end{array}$ \\
\hline
\end{tabular}

overcomes barriers to attending separate services. ${ }^{13} \mathrm{~A}$ collaborative approach between midwives, Aboriginal Health Workers (AHWs) and doctors, all providing consistent advice and support, will reinforce the importance of cessation. The credibility of medical practitioners may be a significant motivating factor for some women. In cases where midwives provide much of the care, the close relationship and frequent contact allows ongoing support. AHWs' cultural knowledge and strong links with local families will enhance implementation of cessation support. ${ }^{14}$ In a survey of Indigenous women, over $70 \%$ of women felt that support from these professionals was likely to be helpful. ${ }^{17}$

\section{Involve other members of the community}

The high prevalence of smoking in Indigenous communities has resulted in smoking being "normalised" as a socially acceptable behaviour, with frequent triggers to smoke and cigarettes being readily available.9,16,18 Smoking is important in social relationships, and cessation can lead to feelings of isolation. ${ }^{18,19}$ Supportive environments for quitting have aided cessation among Indigenous ex-smokers. ${ }^{10}$ Trialling interventions that incorporate mechanisms to provide a supportive, processation environment, such as involving household members in supporting women, peer support groups and whole community interventions should be further explored. $^{20}$

\section{Address relapse}

Interventions that incorporate strategies to prevent smoking relapse result in fewer women relapsing in late pregnancy. ${ }^{1}$ Up to $80 \%$ of women who quit during pregnancy relapse within 1 year. ${ }^{21}$ Specific relapse prevention support should be provided during pregnancy and postpartum, including information about the effects of environmental tobacco smoke on the baby, support to make a smoke-free home and support for household members to quit smoking. ${ }^{21}$ Relapse prevention strategies have not been examined among Indigenous women and should be included in future trials.

\section{Use contingency-based financial rewards}

Systematic reviews of antenatal smoking cessation interventions have found that financial rewards contingent on successful smoking abstinence are significantly more effective than other interventions. ${ }^{1}$ However, their efficacy with Indigenous women has not been tested. Australian surveys indicate that contingency-based rewards are considered likely to be helpful by over $90 \%$ of Indigenous women and $83 \%$ of their antenatal providers. ${ }^{17,22}$ This approach should be further explored with Indigenous women.

\section{Other substances}

Surveys of pregnant Indigenous women found that tobacco smokers were more than three times more likely than non-smokers to report cannabis or alcohol use, both of which are risk factors for continued smoking. ${ }^{17}$ Given the known negative impact of these substances on birth outcomes and the interaction between their use and use of tobacco, interventions should include explicit assessment of other substance use, with support to address these if required. ${ }^{11}$

\section{Training providers}

A lack of protocols and poor smoking cessation support skills have been identified as barriers to providing cessation support to pregnant Indigenous women. ${ }^{12}$ Well defined protocols detailing specific procedures, and the role of each provider, may assist in increasing provision of support in routine care. ${ }^{13}$ Training should cover skills in smoking cessation support, supportive communication and using protocols, as well as recording women's smoking status, cessation behaviour and support provided, to facilitate consistent advice from all team members.

\section{Possible challenges}

Conducting complex behavioural intervention trials is difficult. Potential challenges include: 


\section{Random allocation}

As smoking cessation support is provided at both the service and individual level, randomisation at the individual level is inappropriate as contamination between groups is likely. Cluster randomised controlled trials with randomisation of dispersed services may reduce this problem but require larger sample sizes and more participating services, increasing costs and logistics challenges. As services and communities may not be willing to be randomly allocated to "usual care", it may be more appropriate to undertake a head-to-head comparison of two approaches considered likely to be effective. $^{23}$

\section{Adherence to protocols}

Poor adherence to intervention protocols may occur as a result of unsuitable intervention requirements, inadequate staff training, high staff turnover and lack of systems to support the intervention. Smoking among AHWs has also been identified as a potential barrier to implementation and would need to be addressed as part of the intervention. $^{14,16}$ Strong organisational support for the implementation and evaluation of strategies is critical to supporting adherence. Collaborative development of the intervention and study design with Indigenous services and pilot studies to assess acceptability and feasibility of the research will help successful implementation.

\section{Conclusions}

Given the importance of finding effective strategies to decrease smoking among pregnant Indigenous women, and the current lack of evidence to guide this process, there is an urgent need for rigorous studies to test innovative approaches. While there are many challenges in this research, these may be managed with existing methods for testing complex interventions in diverse settings. ${ }^{24}$ Without an evidence base, we risk implementing ineffective strategies, failing to improve outcomes and wasting scarce resources.

Competing interests: No relevant disclosures.

Provenance: Not commissioned; externally peer reviewed.

1 Lumley J, Chamberlain C, Dowswell T, et al. Interventions for promoting smoking cessation during pregnancy. Cochrane Database Syst Rev 2009; (3): CD001055.

2 Li Z, Zeki R, Hilder L, Sullivan EA. Australia's mothers and babies 2010 Canberra: Australian Institute of Health and Welfare, 2012. (AlHW Cat. No. PER 57; Perinatal Statistics Series No. 27.) http://www.aihw.gov.au/publicationdetail/?id=60129542376 (accessed May 2013).

3 Chan A, Keane RJ, Robinson JS. The contribution of maternal smoking to preterm birth, small for gestational age and low birthweight among Aboriginal and non-Aboriginal births in South Australia. Med J Aust 2001; 174: 389-393.
4 Carson KV, Brinn MP, Peters M, et al. Interventions for smoking cessation in Indigenous populations. Cochrane Database Syst Rev 2012; (1): CD009046.

5 DiGiacomo M, Davidson PM, Abbott PA, et al. Smoking cessation in Indigenous populations of Australia, New Zealand, Canada, and the United States: elements of effective interventions. Int J Environ Res Public Health 2011; 8 : 388-410.

6 Cochrane Effective Practice and Organisation of Care Review Group. Suggested risk of bias criteria for EPOC reviews. Ottawa: Cochrane Collaboration, nd. http://epoc.cochrane.org/sites/epoc.cochrane.org/files/uploads/ Suggested\%20risk\%20of\%20bias\%20criteria\%20for\%20EPOC\%20review s.pdf (accessed Oct 2012).

7 Patten CA, Windsor RA, Renner CC, et al. Feasibility of a tobacco cessation intervention for pregnant Alaska Native women. Nicotine Tob Res 2010; 12 79-87.

8 Eades SJ, Sanson-Fisher RW, Wenitong M, et al. An intensive smoking intervention for pregnant Aboriginal and Torres Strait Islander women: a randomised controlled trial. Med J Aust 2012; 197: 42-46.

9 Wood L, France K, Hunt K, et al. Indigenous women and smoking during pregnancy: knowledge, cultural contexts and barriers to cessation. Soc Sci Med 2008; 66: 2378-2389.

10 Bond C, Brough M, Spurling G, Hayman N. "It had to be my choice" Indigenous smoking cessation and negotiations of risk, resistance and resilience. Health, Risk \& Society 2012; 14: 565-581. doi: 10.1080/13698575.2012.701274.

11 NSW Department of Health. National clinical guidelines for the management of drug use during pregnancy, birth and early development years of the newborn. Sydney: NSW DoH, 2006. http://www0.health.nsw.gov.au/pubs/ 2006/ncg_druguse.html (accessed May 2013).

12 Passey ME, D'Este CA, Sanson-Fisher RW. Knowledge, attitudes and other factors associated with assessment of tobacco smoking among pregnant Aboriginal women by health care providers: a cross-sectional survey. BMC Public Health 2012; 12: 165.

13 Baxter S, Everson-Hock E, Messina J, et al. Factors relating to the uptake of interventions for smoking cessation among pregnant women: a systematic review and qualitative synthesis. Nicotine Tob Res 2010; 12: 685-694.

14 Thompson M, Robertson J, Clough A. A review of the barriers preventing Indigenous Health Workers delivering tobacco interventions to their communities. Aust N Z J Public Health 2011; 35: 47-53.

15 Passey ME, D’Este CA, Stirling JM, Sanson-Fisher RW. Factors associated with antenatal smoking among Aboriginal and Torres Strait Islander women in two jurisdictions. Drug Alcohol Rev 2012; 31: 608-616.

16 Gould GS, Munn J, Watters T, et al. Knowledge and views about maternal tobacco smoking and barriers for cessation in Aboriginal and Torres Strait Islanders: a systematic review and meta-ethnography. Nicotine Tob Res 2013; 15: 863-874.

17 Passey M, Sanson-Fisher R, Gale J, Stirling J. Tobacco, alcohol and cannabis during pregnancy: views of pregnant Aboriginal women in NSW and the ACT. Report for the Australian Government Department of Health and Ageing. Lismore: University of Sydney, 2011.

18 Johnston V, Thomas DP. Smoking behaviours in a remote Australian Indigenous community: the influence of family and other factors. Soc Sci Med 2008; 67: 1708-1716.

19 Passey ME, Gale JT, Sanson-Fisher RW. "It's almost expected": rural Australian Aboriginal women's reflections on smoking initiation and maintenance: a qualitative study. BMC Womens Health 2011; 11: 55.

20 Secker-Walker RH, Gnich W, Platt S, Lancaster T. Community interventions for reducing smoking among adults. Cochrane Database Syst Rev 2002; (3): CD001745.

21 Mullen PD. How can more smoking suspension during pregnancy become lifelong abstinence? Lessons learned about predictors, interventions, and gaps in our accumulated knowledge. Nicotine Tob Res 2004; 6 Suppl 2: S217-S238.

22 Passey M, Sanson-Fisher R, Gale J, Stirling J. Antenatal care for Aboriginal women: views of NSW and ACT service providers regarding tobacco, alcohol and cannabis use. Report for the Australian Government Department of Health and Ageing. Lismore: University of Sydney, 2010.

23 Sox HC, Goodman SN. The methods of comparative effectiveness research. Annu Rev Public Health 2012; 33: 425-445.

24 Hawe P, Shiell A, Riley T. Complex interventions: how "out of control" can a randomised controlled trial be? BMJ 2004; 328: 1561-1563.

\section{When too much MJA and MJA InSight is never enough.}

Follow us on Twitter (@theMJA) and like us on Facebook (https://www.facebook.com/theMJA)

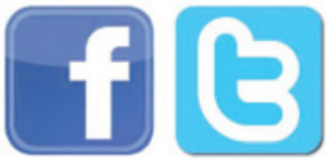

